\title{
Tracking Area Boundary-aware Protocol for Pseudo Stochastic Mobility Prediction in LTE Networks
}

\author{
Vincent Omollo Nyangaresi \\ School of Information Sciences and Technology, Kisii University, 40200 - Kenya \\ E-mail: vincent@kisiiuniversity.ac.ke
}

\section{Silvance O. Abeka}

School of Informatics \& Innovative Systems, Jaramogi Oginga Odinga University of Science \& Technology - Kenya E-mail: silvancea@gmail.com

\author{
Anthony J. Rodrigues \\ School of Informatics \& Innovative Systems, Jaramogi Oginga Odinga University of Science \& Technology - Kenya. \\ E-mail: tonyr@jooust.ac.ke
}

Received: 29 December 2019; Accepted: 14 February 2020; Published: 08 October 2020

\begin{abstract}
Accurate mobility prediction enables efficient and faster paging services in these networks. This in turn facilitates the attainment of higher bandwidths and execution of activities such as handovers at low latencies. The conventional mobility prediction models operate on unrealistic assumptions that make them unsuitable for cellular network mobile station tracking. For instance, the Feynman-Verlet, first order kinetic model and Random Waypoint assume that mobile phones move with constant velocity while Manhattan, Freeway, city area, street unit, obstacle mobility, and pathway mobility postulate that mobile station movement is restricted along certain paths. In addition, obstacle mobility model speculate that the mobile station signal is completely absorbed by an obstacle while random walk, random waypoint, Markovian random walk, random direction, shortest path model, normal walk, and smooth random assume that a mobile station can move in any direction. Moreover, the greatest challenge of the random direction model is the requirement that a border behavior model be specified for the reaction of mobile stations reaching the simulation area boundary. In this paper, a protocol that addresses the border behavior problem is developed. This protocol is shown to detect when the subscriber has moved out of the current tracking area, which is crucial during handovers.
\end{abstract}

Index Terms: Boundary detection, Mobility prediction, modeling, LTE, latency.

\section{Introduction}

The field of mobile communication systems has tremendously shifted starting from the second generation Global System for Mobile Communications (2G/GSM), third generation Universal Mobile Telecommunication Systems (3G/UMTS), the fourth generation Long Term Evolution (4G/LTE) systems to the fifth generation (5G) networks.In cellular networks, mobility prediction is concerned with envisaging of the mobile station's next movement. By accurately employing the predicted movement, the network is capable of attaining enhanced resource allocation and reservations, better assignment of cells to location areas, more efficient paging, and call admission control.

The ultimate goal of mobility prediction models is to make an attempt in imitating the movement of real mobile stations (MSs), which are characterized by the change of speed and direction with time [1]. This allows the network to track the current location of the subscribers. In so doing these mobility models permit voice calls, short messaging services (SMS), general packet radio services (GPRS) and other mobile phone services to be delivered to the subscribers.

According to [2], mobility prediction models are significant in the provision and maintenance of communication with a mobile user at any given point in time. This is particularly true now that there has been a growing trend of the convergence of numerous financial services such as banking applications with mobile communication services. However, with the swift growth in the number of mobile subscribers globally, mobility prediction has emerged as one 
of the most important and challenging tasks for mobile communication systems.

Since majority of subscribers experience random atomic movements within the coverage area, random waypoint and random direction mobility are popular in depicting such movements. However, both models assume that subscribers can move anywhere within the coverage area. This is unrealistic as physical obstacles such as mountains and water bodies can impede such random mobility. In addition, random waypoint model ignores direction changes while random direction requires the definition of border behavior when the subscriber approaches the cellular network boundary. The contributions of this paper include the following:

1) We develop a protocol that combines the random waypoint model and random direction model that is demonstrated to have realistic mobility prediction.

2) We design and develop the boundary behavior of user equipment (UE) to address the border behavior problem in random direction model.

3) We demonstrate through simulations that when the cumulative density function (CDF) of the received signal level is higher than the threshold signal level, $P_{\min }$, the UE is within the coverage area. On the other hand, if the CDF is lower than $P_{\min }$, then the UE is at the cluster boundary.

The rest of this paper is organized as follows: section II provides related work in the area of mobility prediction while part III gives the methodology adopted to develop our protocol. Section IV presents the results obtained from the simulations as well as the discussion while part, section $\mathrm{V}$ concludes the paper.

\section{Related Work}

A number of studies have been carried out in mobile station prediction and as such, many mobility predictions have been developed. Due to the significance of mobility prediction, several studies have been conducted and their results have led to the development of a number of mobility prediction models such as random waypoint, random walk, random Markovian walk, random direction mobility, smooth random mobility, cell-residence-time-based, GaussMarkov, Fluid Flow, normal walk, shortest path, activity-based, pursue mobility, nomadic community mobility, reference point group mobility(in-place mobility, overlap mobility, convention mobility), Manhattan grid, pathway mobility, obstacle mobility, Freeway mobility, Street Unit, Street Pattern Tracing, mobility vector, gravity models, city section mobility, city area mobility, First order Kinetic, Feynman-Verlet, Semi-Hidden Markov, Autoregressive (AR), and Global Mobility among others[3].

In obstacle mobility model, mobile station mobility is depicted by taking into consideration real-life scenarios such as the fact that people move towards specific destinations rather than randomly choosing some destinations; obstacles such as buildings, parks or rivers can block people's movements as well hinder signal propagation; and that people do not walk along random directions but along pathways and select shortest paths [4]. In street unit model, the mobile station is permitted to move on a rectangular, Manhattan grid only, where the grid depicts the street pattern of suburban or urban areas [5] while city area mobility model is utilized to describe mobile station mobility and traffic behaviour within a city area environment. The random walk model is an individual mobility model that is memory-less since it does not retain knowledge related to its past speed and direction [3] while [6] discuss the random waypoint model as a simple stochastic model in which a mobile station moves on a restricted continuous plane from its current position to a new location by randomly choosing its destination coordinates, its speed of movement, and the amount of time that it will pause on arriving at the destination.

The Markovian random walk model is modified form of the random walk model that utilizes Markov chains to describe the mobile station movement, and it introduces memory in the movement behavior of the mobile station [7]. Random direction model is described by [8] as a model in which the UE randomly selects a direction from a given interval and moves in that direction. Another mobility prediction model is the shortest path model in which an UE follows the shortest path measured by the number of cells passed through, from source to destination [9].

The normal walk model is straight-oriented mobility model which assumes that a mobile station moves in unit steps on a Euclidean plane [10]. The smooth random mobility model is an enhanced random mobility model that makes the movement trace of individual mobile stations more realistic than common approaches for random movement [11]. Microscopic models depict the movement of a single mobile station by its space and speed coordinates at a given time $t$, and the goal here is to obtain a very detailed representation for one entity within the network coverage area. Such models include Street Unit Models and Street Pattern Tracing Models [12]. On the other hand, mesoscopic models depict the homogenized movement behaviour of several mobile stations instead of only one. As pointed out by [13], the mobile users shift as groups (hence group models). Examples of these models include Reference Point Group Mobility (In-Place Mobility, Overlap Mobility, and Convention Mobility) and Mobility Vector model.

Macroscopic models focus is on density, mean speed, speed variance, and traffic flow of vehicles. Examples of these models include fluid flow models, gravity models and the random walk models [14]. In reference point group model, each group has a center that can be either a logical center or a group leader mobile station. Here, each group is made up of one leader and a number of members [15]. Colum mobility model is described by [16] as being ideal for 
mimicking a set of mobile stations such as robots moving in a certain fixed direction while [17] designate pursue mobility model as being a model in which the pursuer mobile stations direct their velocity towards the position of the targeted mobile station in an attempt to intercept it. Regarding nomadic community mobility model, [18] expound that in this model, the entire group of mobile stations shifts randomly from one location to another and the reference point of each mobile station is established based on the common movement of this group.

In [19], the authors discuss that in activity-based models, once the location for the next activity has been determined, the route from the current location to this activity location is computed in terms of cells traversed. In cellresidence-time-based model, the idea here is to establish the connection time spent by a mobile station within one location. According to [20], this requires the tracing of the movement of individual users. The authors in [21] discuss that in pathway mobility model, initially, the UEs are randomly positioned on the edges of the graph and then for each mobile station, a destination is selected arbitrarily and the mobile station moves towards this destination via the shortest path along the edges.

\section{Methodology}

The developed protocol employed atomic subscriber movements and hence the mobility prediction model adopted was microscopic in scope. On mobility pattern, the protocol utilized pseudo-stochastic movements which were depicted by the boundary -aware combination of the random waypoint and random direction mobility prediction models. The random waypoint was chosen owing to its ability to depict motion waypoints, velocity and pause time while the random direction mobility model was selected because of its ability to incorporate direction changes after every waypoint. The random direction model was modified to address its border behavior specification problem by the incorporation of a border behavior to specify the reaction of mobile stations reaching the simulation area boundary, which was treated as the simulated obstacle.

This was achieved by specifying the threshold received power, $P_{\min }$ and computing the CDF to determine the probability the received signal may be higher or lower than this threshold. Here, when the CDF is higher than $P_{\min }$, the UE is within the coverage area. On the other hand, if the CDF is lower than $P_{\text {min }}$, then the UE is on the cluster boundary. When this happens, the neuro-fuzzy algorithm is invoked to initiate handover request to the target cell.

In the developed protocol, upon reaching the cluster boundary, only the UE movement is blocked by the boundary (treated as an obstacle in this case) and the signals can still reach their intended destination. Essentially, the obstacle forced the mobile station to bounce off and look for alternative paths to the destination. Here, an UE enters and exits with equal probability the cellular network coverage area at arbitrary selected point $P_{i}$ and $P_{o}$ on the circle enclosing the coverage area respectively, as shown in Fig. 1 . The implication is that in Fig. 1 both $\theta_{i}$ and $\theta_{o}$ are uniformly distributed in $[0,2 \pi]$ and $\theta_{n}=\left|\theta_{i}-\theta_{o}\right|$. The probability density functions (PDFs) of the entry and exit locations are equal to $\frac{1}{2 \pi}$.

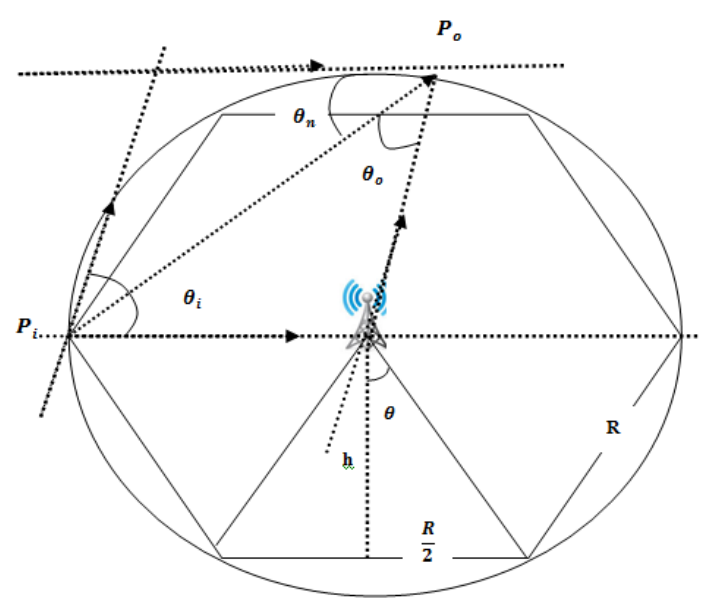

Fig.1. Cellular Network Coverage Area Modeling

To model UE mobility pattern in the cellular network using the developed pseudo-stochastic mobility tracking protocol, the cellular network surface area was first estimated. To accomplish this, the hexagonal cellular network is divided into 6 isosceles triangles each of height $h$ as shown in Fig. 1. The base of each of these triangles is $\mathrm{R}$ and the sum of all exterior angles is equal to 360 degrees and hence $\theta=\frac{360}{12}=30$ degrees.Mathematically:

$$
\text { tangent } \theta=\frac{\text { opposite }}{\text { Adjacent }}=\frac{\frac{R}{2}}{h} ; \tan 30^{\circ}=\frac{R}{2 h}
$$


But $\tan 30^{\circ}=0.5773=\frac{\sqrt{3}}{3}$ and Hence (1) assumes the form given in (2):

$$
\frac{\sqrt{3}}{3}=\frac{R}{2 h} ; h=\frac{3 R}{2 \sqrt{3}}
$$

The area of a triangle is $\frac{1}{2} b h$, where $b$ is the base and $h$ is the height. From Fig. 1 , the base is $\frac{R}{2}$ and hence the area is expressed by (3):

$$
\text { Trianglearea }=\frac{1}{2} * \frac{R}{2} * \frac{3 R}{2 \sqrt{3}}=\frac{3 R^{2}}{4 \sqrt{3}}
$$

The hexagon consists of six triangles and hence its area assumes the form given in (4):

$$
\text { Hexagonalarea, } \begin{aligned}
A_{\text {hex }} & =6 * \text { Trianglearea } \\
& =6 * \frac{3 R^{2}}{4 \sqrt{3}}=\frac{3 \sqrt{3}}{2} R^{2}
\end{aligned}
$$

From (4), the area of the hexagonal cellular network is $2.598 R^{2}$ and is the value employed for the computation of the various handover figures of merit as discussed in the next sub-section. The algorithm for the pseudo-stochastic mobility tracking protocol required input parameters that included propagation environment factors, threshold received power, the reference distance, transmitter-UE distance, and transmitted power as shown in Fig. 2.

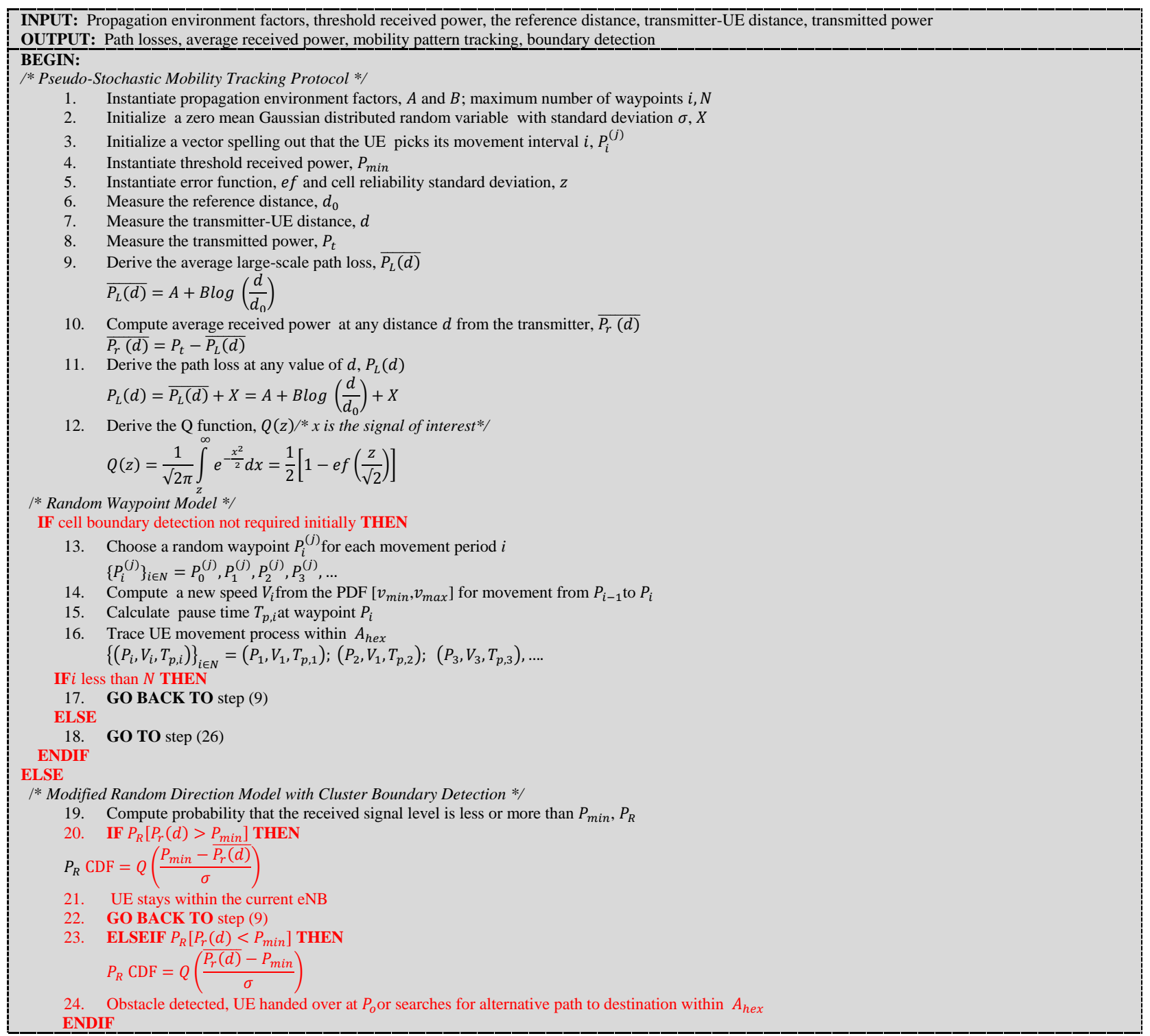




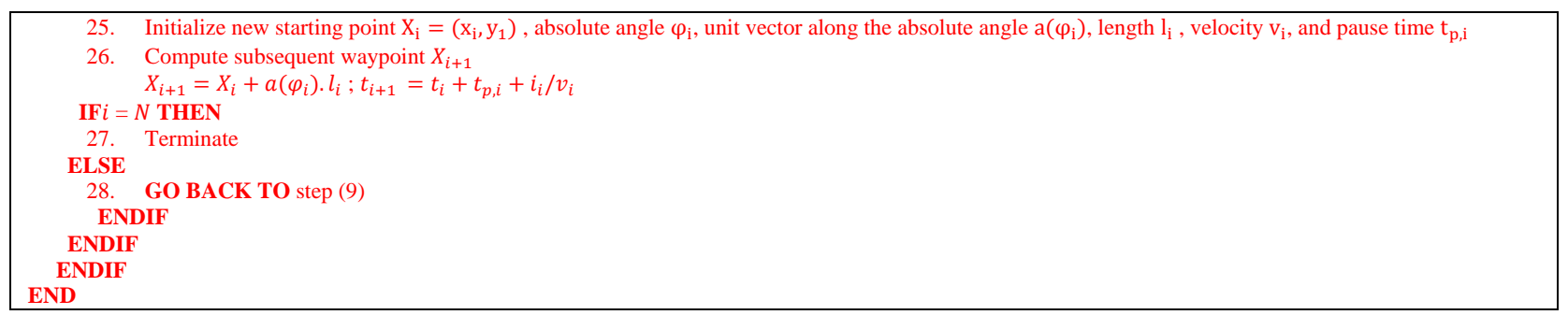

Fig.2. Algorithm for Pseudo-Stochastic Mobility Tracking Protocol

Thereafter, a number of computations were executed which comprised of derive the average large-scale path loss (step-9), average received power at any distance $d$ from the transmitter (step-10), path loss at any value of $d$ (step-11), derivation of the $\mathrm{Q}$ function based on the signal of interest and the cell reliability standard deviation (step-12), selection of random waypoint for each movement period (step-13), computation of a new velocity for the next motion waypoint (step-14), calculation of the pause time at the new waypoint (step-15), computation of the probability that the received signal level is less or more than the threshold value (step-19).

On condition that the value in step (19) is more than the threshold value, the UE was still within the current eNB. This was accomplished through invoking the random direction mobility model in which the current location is initalized as a new starting point and the new mobility parameters such as absolute angle from the range $[0,2 \pi]$, unit vector along the absolute angle, new waypoint length, new velocity and pause time are also initialized (step-25). Thereafter, the subsequent UE location is computed (step-26) and the process repeats itself (step 28). In case the subscriber is handed over to the new tracking area, then the PDF of using any location of the circular radio frequency coverage area is $\frac{1}{2 \pi}$ as already elaborated. Fig. 3 models these pseudo-stochastic mobility tracking protocol procedures.

The output of this algorithm include path losses, average received power, mobility pattern tracking, and boundary detection, all of which helped track the mobility of the UE within the coverage area depicted in Fig. 2 above. The modeling described in this section was then converted into source codes which were then executed in Python programming language to yield the results discussed in section IV below.

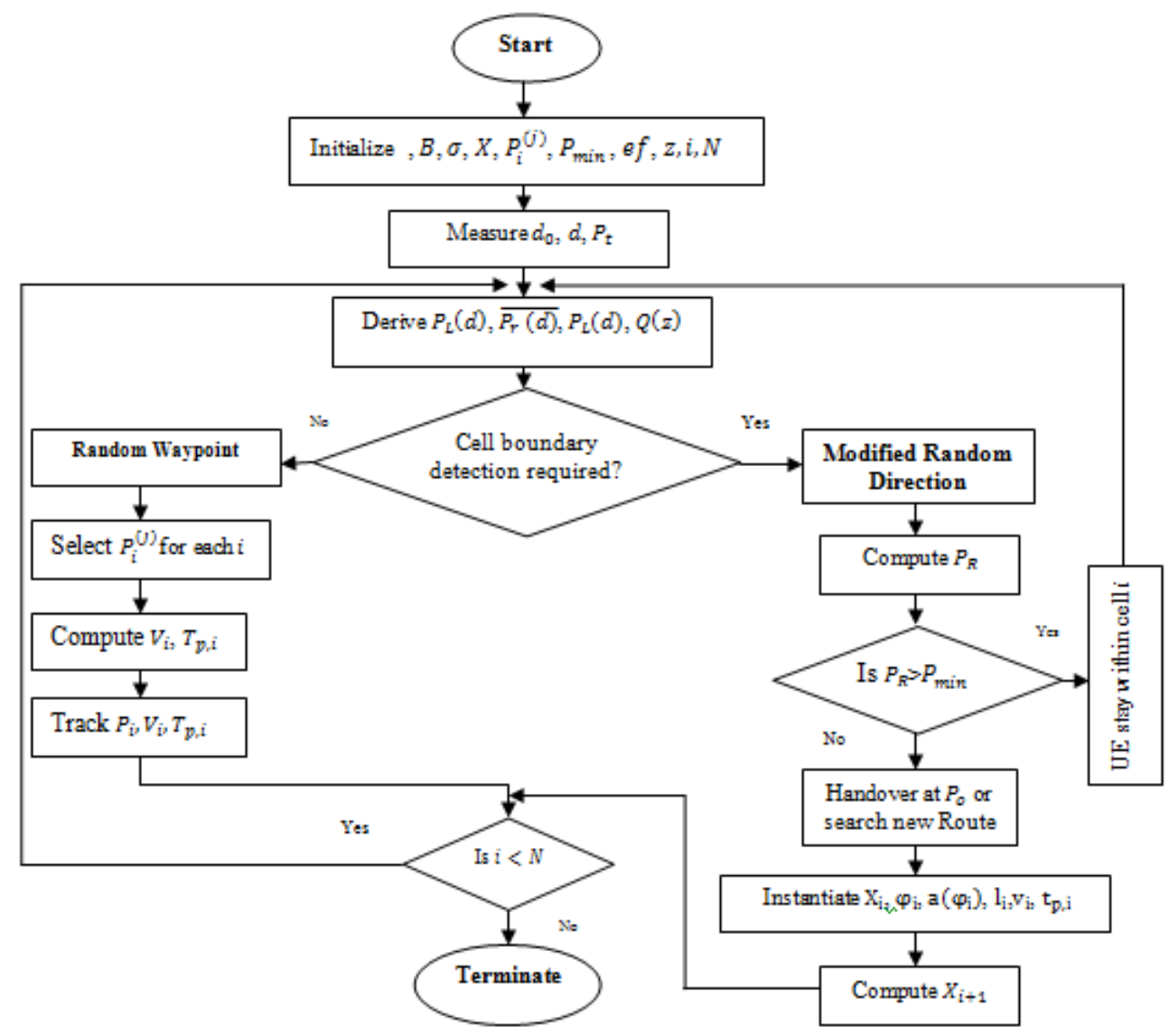

Fig.3. Modeling Pseudo-Stochastic Mobility Tracking Protocol 
However, if this value is less than the threshold value, then the UE is at the cluster boundary and should either be handed over or search for a new route to the destination (step-18).

\section{Results}

The developed protocol combined both random direction mobility model and random waypoint mobility model into a single model. At the onset of subscriber mobility, random waypoint mobility was initiated but upon boundary detection, the modified random direction model was activated. As shown in Fig. 4, the UE mobility is characterized by random selection of destination coordinates, velocity and pause time in accordance with the random waypoint model. This is the stochastic mobility which represents the randomness of mobility.

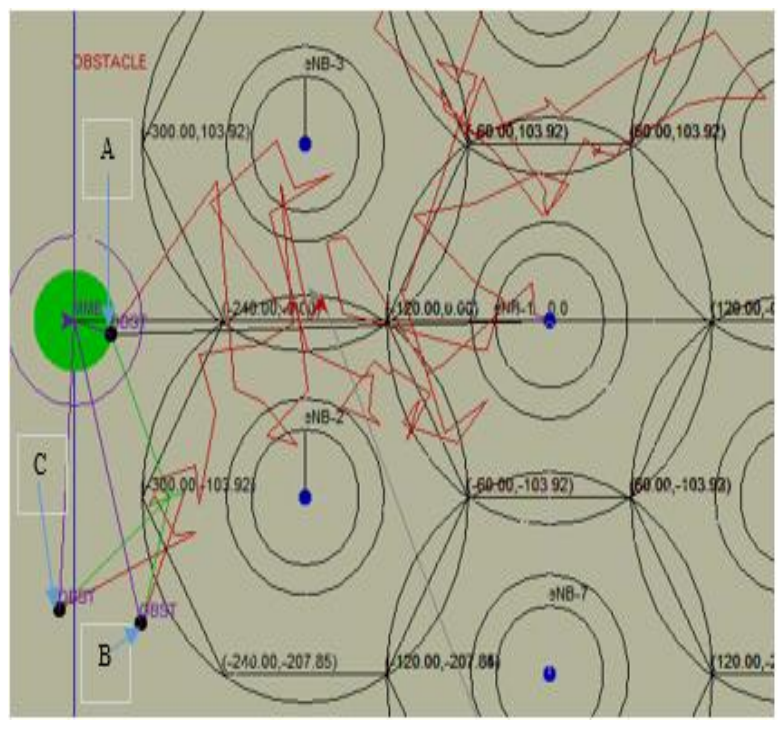

Fig.4. Pseudo-Stochastic Mobility

The UE adopts the random waypoint model so long as the received power $\operatorname{Pr}(d)$ is less than the threshold power, $P_{\min }$. The simulations that were carried out yielded $-182 \mathrm{~dB}$ as $P_{\min }$, which was the received power at the onset of HPHR. As Fig. 4demonstrates, during the observation period, three boundary locations were detected.

As detailed in Fig. 5, upon the reduction of the $\operatorname{Pr}(d)$ below $P_{\min \text {, an }}$ UE shifted from the random waypoint model to the modified random direction model in which the new starting point $X_{i}$, absolute angle $\varphi_{i}$, unit vector along the absolute angle $\mathrm{a}\left(\varphi_{\mathrm{i}}\right)$, length $\mathrm{l}_{\mathrm{i}}$, velocity $\mathrm{v}_{\mathrm{i}}$, and pause time $\mathrm{t}_{\mathrm{p}, \mathrm{i}}$ are computed at the onset of the movement process.

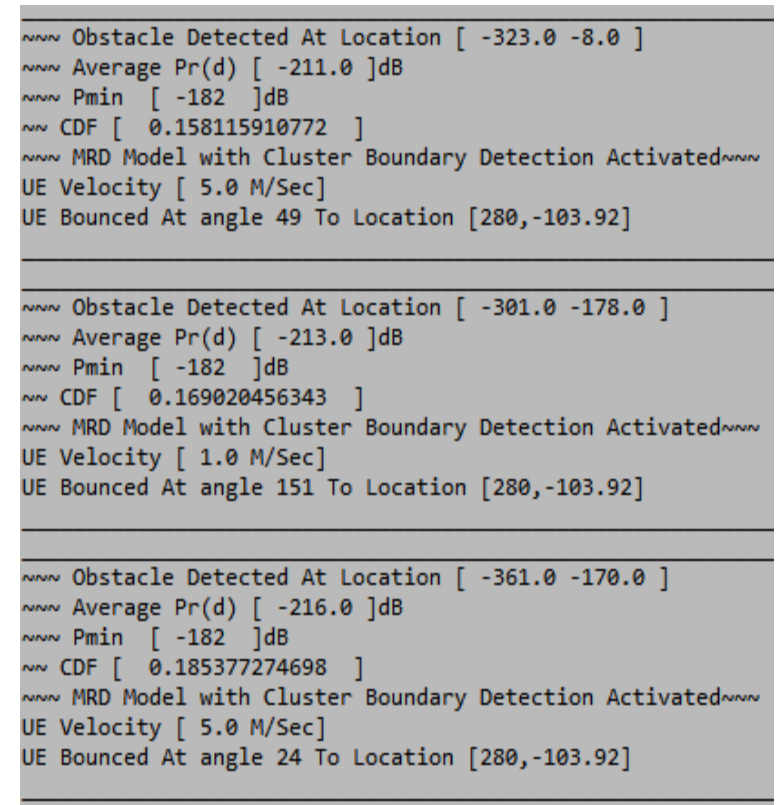

Fig.5. Boundary Detection 
In the modified random direction (MRD) model, the values for the direction were taken from a uniform distribution on the interval $[0,2 \pi]$ while the values for the velocity followed a uniform distribution. As shown in Fig. 5, the first boundary location was detected (OBST) near the MME at $\mathbf{A}(-323.0-8.0)$ at which the $\operatorname{Pr}(d)$ was $-211.0 \mathrm{~dB}$ while the CDF was approximately 0.16. Upon boundary detection, an UE was redirected at absolute angle $\varphi_{\mathrm{i}}=49^{0}$ with $\mathrm{v}_{\mathrm{i}}=5.0 \mathrm{M} / \mathrm{Sec}$ and new starting point $\mathrm{X}_{\mathrm{i}}=(280,-103.92)$.

The second boundary was detected at $\mathbf{B}(-301.0-178.0)$ at which the $\operatorname{Pr}(d)$ was $-213.0 \mathrm{~dB}$ while the CDF was approximately 0.17 . Upon boundary detection, an UE was redirected at absolute angle $\varphi_{\mathrm{i}}=151^{0}$ with $\mathrm{v}_{\mathrm{i}}=1.0 \mathrm{M} / \mathrm{SeC}$ and new starting point $\mathrm{X}_{\mathrm{i}}=(280,-103.92)$. The third boundary location was detected at $\mathbf{C}(-361.0-170.0)$ at which the $\operatorname{Pr}(d)$ was $-216.0 \mathrm{~dB}$ while the CDF was approximately 0.19 . Upon boundary detection, an UE was redirected at absolute angle $\varphi_{\mathrm{i}}=24^{0}$ with $\mathrm{v}_{\mathrm{i}}=5.0 \mathrm{M} / \mathrm{Sec}$ and new starting point $\mathrm{X}_{\mathrm{i}}=(280,-103.92)$. In Table 1 , the variations of CDF against the received signal level $\mathrm{P}_{(\mathrm{rd})}$ at a constant $\mathrm{P}_{\min }$ value of $-182 \mathrm{~dB}$ are given.

Table 1. Variation of CDF Against P (rd)

\begin{tabular}{|c|c|c|c|}
\hline Simulation Iterations & $\mathbf{P}_{\min }(\mathbf{d B})$ & $\mathbf{P}_{(\mathbf{r d})}(\mathbf{d B})$ & CDF Value \\
\hline 1 & -182 & -211 & 0.158 \\
\hline 2 & -182 & -214 & 0.174 \\
\hline 3 & -182 & -217 & 0.191 \\
\hline 5 & -182 & -212 & 0.164 \\
\hline 6 & -182 & -213 & 0.169 \\
\hdashline 7 & -182 & -215 & 0.180 \\
\hline$\ldots \ldots$ & -182 & -218 & 0.196 \\
\hline
\end{tabular}

As shown in Table 1, the value of $\mathrm{P}_{\min }$ was maintained at $-182 \mathrm{~dB}$ while the value of received signal levels were measured as the UE traversed the tracking area. The highest $P_{(r d)}$ was $-211 \mathrm{~dB}$ while the least $P_{(r d)}$ Was $-218 \mathrm{~dB}$. Fig. 6 presents a graph of CDF against the received signal levels at various locations within the tracking area.

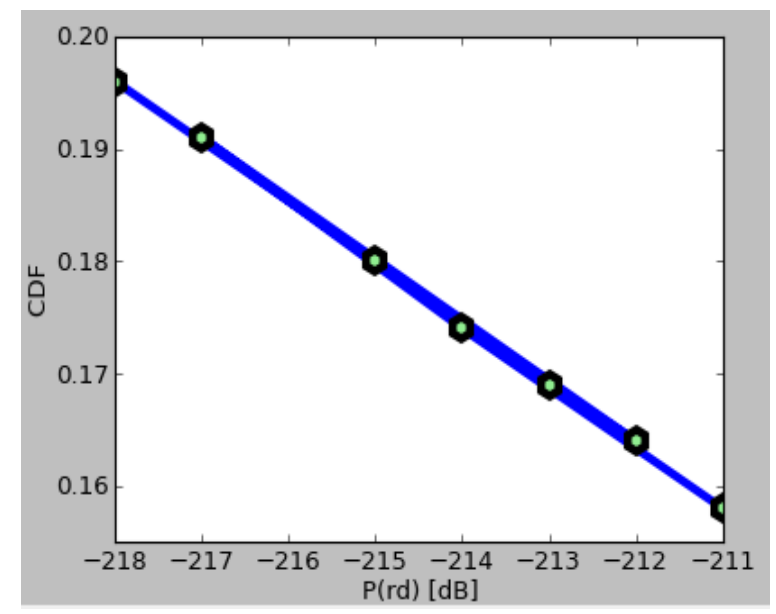

Fig.6. CDF Variations with $\mathrm{P}_{(\mathrm{rd})}$

Fig. 6 demonstrates that graph of CDF against $\mathrm{P}_{(\mathrm{rd})}$ is linear, implying that as the value of $\mathrm{P}_{(\mathrm{rd})}$ is increased, the value of CDF reduces and vice versa. As such, when an UE is located near the eNB, the value of $\mathrm{P}_{(\mathrm{rd})}$ is high and the CDF value if very low and hence the cell boundary is located far away from the eNB. On the other hand, when an UE is further away from the eNB, the value of $\mathrm{P}_{(\mathrm{rd})}$ is very low but the value of CDF is very high, implying that the UE is at the proximity of the cell boundary.

In addition, the developed protocol detected when the UE moved out of the current tracking area to the next tracking area as shown in Fig. 7. As demonstrated here, an UE was detected to be shifting towards the neighbouring tracking area at a location whose coordinates were 73.0, 318.0 at a velocity of 4.0

$\mathrm{M} / \mathrm{Sec}$ and as such, the control of the communication process was transferred to the macro-cell. This is because macro-cells span relatively large coverage areas and as such are better suited to control the communication for UEs that frequently shift from one cluster to another so as to offer seamless connectivity.

Based on the mobility traces of Fig. 4 and Fig. 7, the values of $l_{i}$ were different for diverse UE movements. This is further confirmed by a sample of the mobility vectors depicted in Fig. 8 below. In (a), the UE has a starting $\mathrm{X}_{\mathrm{i}}=$ $(280,255), \varphi_{\mathrm{i}}=19, \mathrm{a}\left(\varphi_{\mathrm{i}}\right)=0.749, l_{i}=17.99, \mathrm{v}_{\mathrm{i}}=5$ and $\mathrm{t}_{\mathrm{p}, \mathrm{i}}=0$. 


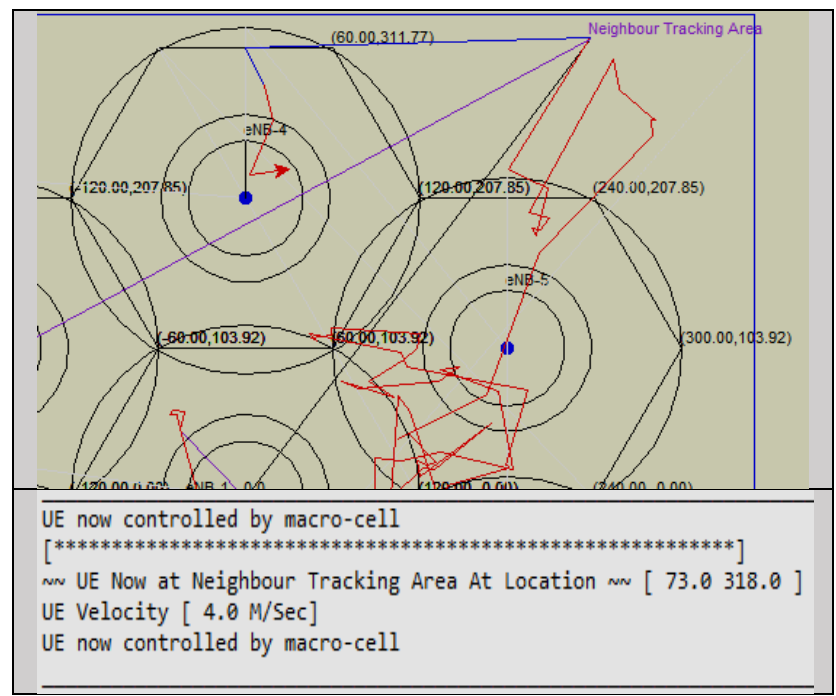

Fig.7. Neighbouring Tracking Area Detection

This mobility was accompanied by the random shifting of the UE mobility vectors, some of which are shown in Table 2.

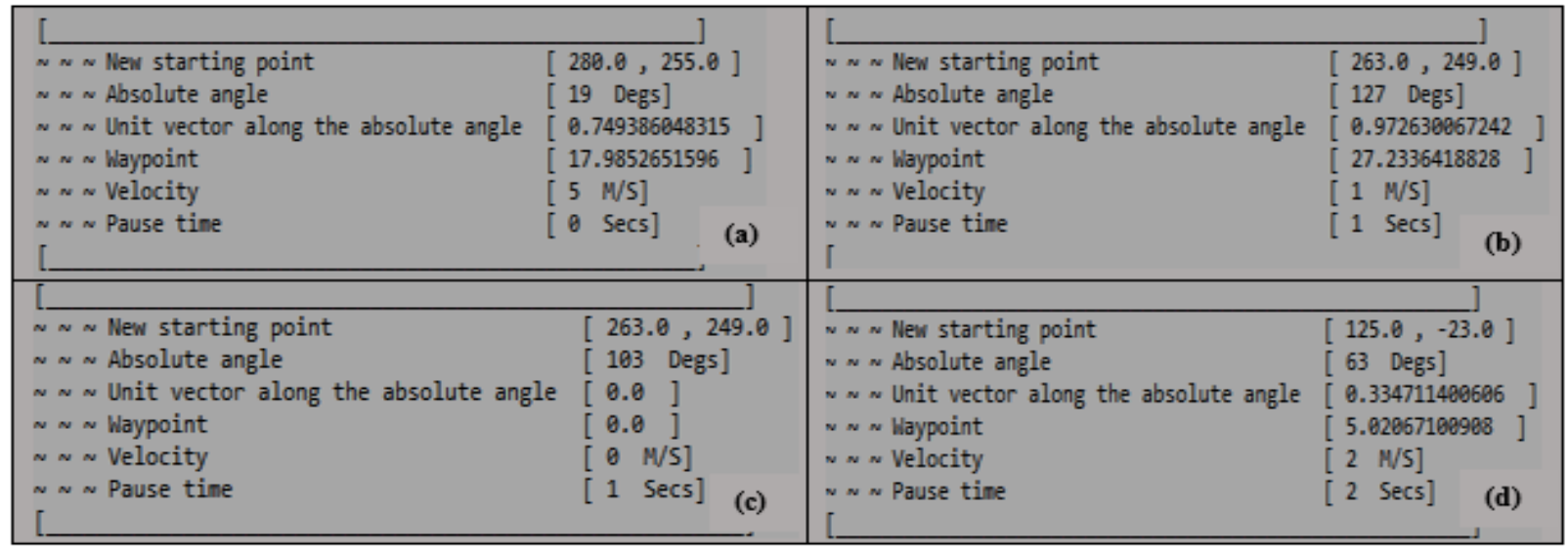

Fig.8. Pseudo-Stochastic Mobility Tracking

The UE X-axis coordinates shifted from a maximum of 280 to the minimum of 92 while the Y-axis coordinates shifted from maximum value $X_{\max }$ of 255 to a minimum $X_{\min }$ of -70 .

Table 2. Tracking Mobility Vectors

\begin{tabular}{|c|c|c|c|c|c|c|}
\hline Simulation Iterations & $\left(\mathbf{x}_{\mathbf{i}}, \mathbf{y}_{\mathbf{1}}\right)$ & $\boldsymbol{\varphi}_{\mathbf{i}}$ & $\mathbf{a}\left(\boldsymbol{\varphi}_{\mathbf{i}}\right)$, & $\mathbf{l}_{\mathbf{i}}$ & $\mathbf{v}_{\mathbf{i}}$, & $\mathbf{t}_{\mathbf{p}, \mathbf{i}}$ \\
\hline 1 & $280.0,255.0$ & 19 & 0.749 & 17.99 & 5 & 0 \\
\hline 2 & $263.0,249.0$ & 127 & 0.973 & 27.234 & 1 & 1 \\
\hline 3 & $263.0,249.0$ & 103 & 0 & 0 & 0 & 1 \\
\hline 4 & $125.0,-23.0$ & 63 & 0.335 & 5.021 & 2 & 2 \\
\hline 5 & $114.0,-70.0$ & 39 & 2.891 & 34.697 & 3 & 1 \\
\hline 6 & $104.0,-39.0$ & 64 & 3.680 & 33.121 & 4 & 1 \\
\hline 7 & $92.0,26.0$ & 135 & 0.353 & 7.776 & 4 & 2 \\
\hline
\end{tabular}

Regarding the absolute angle, its value dropped from the maximum $\boldsymbol{\varphi}_{\max }$ of $135^{\circ}$ to the minimum $\boldsymbol{\varphi}_{\min }$ of $19^{0}$. The unit vector along this absolute angle shifted from a peak value $\mathrm{a}(\boldsymbol{\varphi})_{\text {(max) }}$ of 3.680 to the lowest value $\mathrm{a}(\boldsymbol{\varphi})_{\text {(min) }}$ of 0 . On its part, the waypoints selected peaked $l_{\max }$ value of 34.697 and ditched at the lowest $l_{\min }$ value of 0 . The maximum velocity $v_{\max }$ was 5 while the minimum velocity $v_{\min }$ was 0 when the UE selected 0 waypoint at (c) in Fig. 8. Fig. 9 presents the graphs for the figures of merit shown in Table 2. 

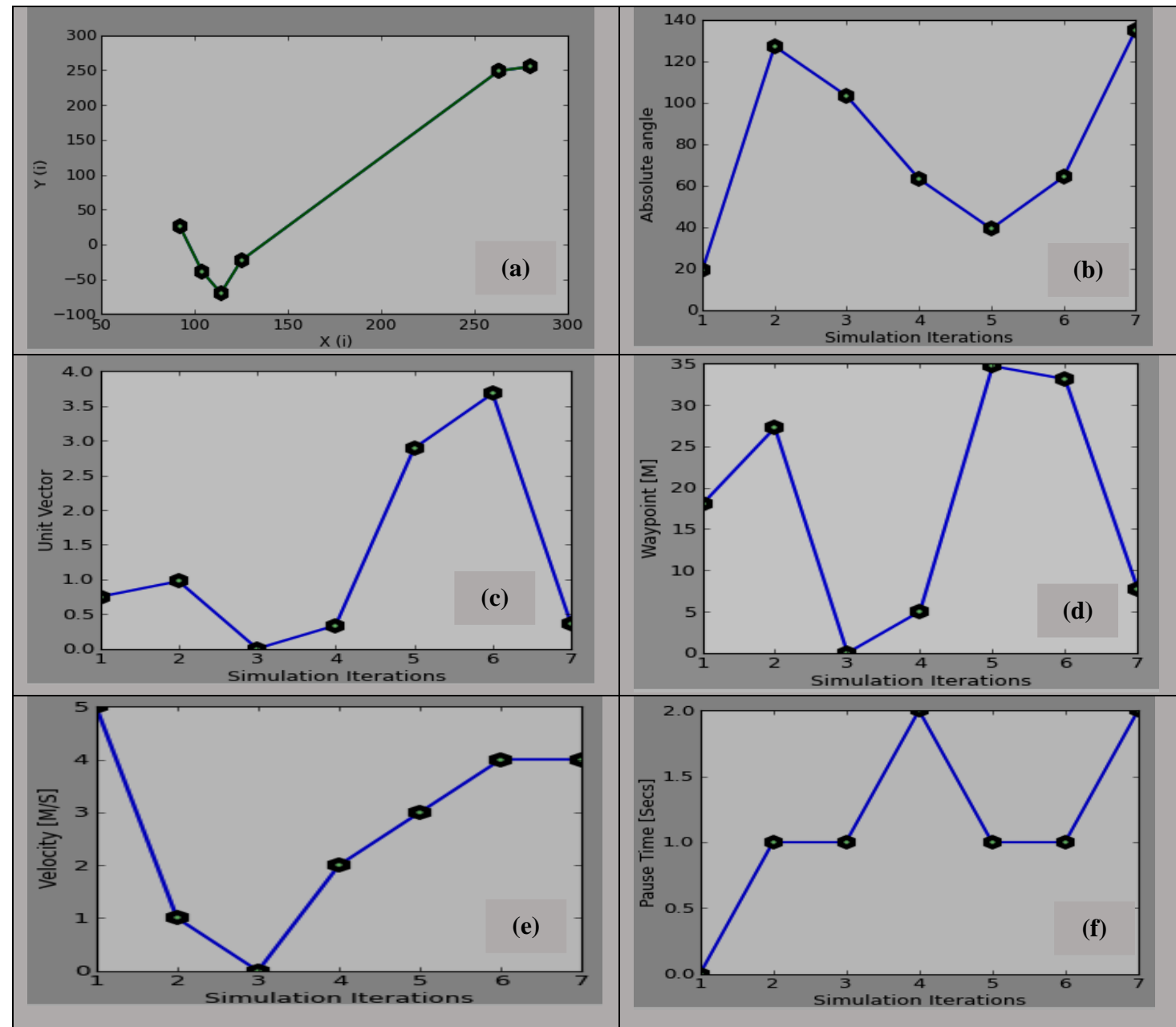

Fig.9. Variations of Mobility Vectors

All the graphs in Fig. 9 demonstrate that the mobility vectors were randomly selected for each UE movement. In Fig. 9 (a), the UE coordinates is observed to be stochastic, similar to the variation of absolute angle (b), unit vector along the absolute angle (c), waypoints (d), velocity (e) and pause time (f).

Fig. 10 compares the pseudo-stochastic mobility with the combined random direction and random waypoint mobility models. As demonstrated in Fig. 10 (a), although the conventional random mobility models define new starting point $\mathrm{X}_{\mathrm{i}}$, random waypoint $P_{i}^{(j)}$ for each movement period $i$, pause time $T_{p, i}$ at waypoint $P_{i}$, absolute angle $\varphi_{\mathrm{i}}$, unit vector along the absolute angle $\mathrm{a}\left(\varphi_{\mathrm{i}}\right)$, and velocity $\mathrm{v}_{\mathrm{i}}$, they both fail to define the boundary behavior upon obstacle detection. As such, the UE is observed to have moved well beyond the cluster boundary. This challenge was addressed in the developed protocol by use of CDF for both $P_{\min }$ and the received signal level and hence was able to define boundary behavior as illustrated in Fig. 10 (b). This is the pseudo-stochastic mobility that this research paper sought to achieve such that the subscriber mobility within the cellular network is able to recognize the presence of obstacles that impede mobility in any direction. In so doing, the subscriber mobility assumes more realistic assumptions. 


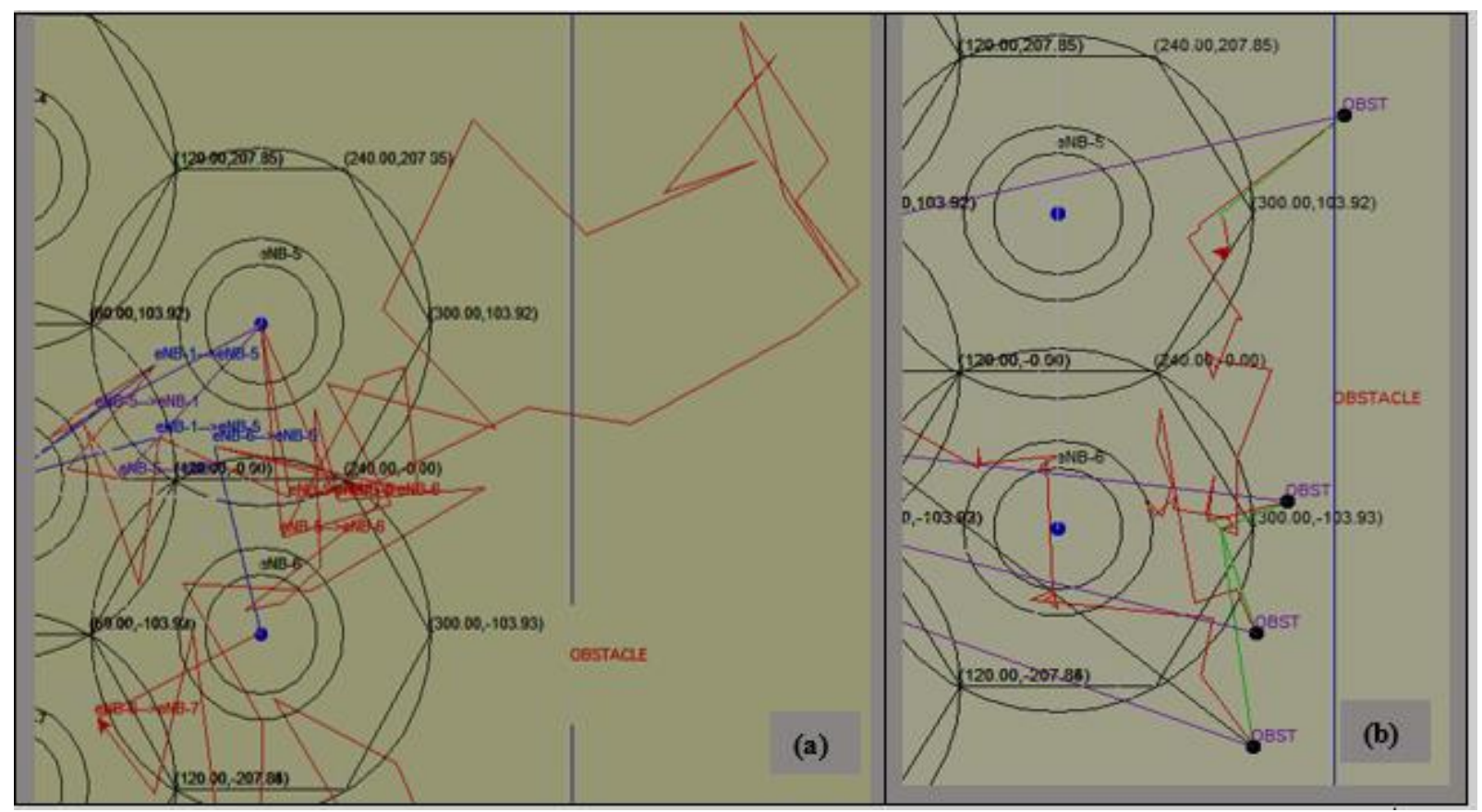

Fig.10. Pseudo-Stochastic Mobility and Random Mobility Models Comparison

\section{Conclusion}

The current random waypoint mobility model fails to consider direction changes after every waypoint. Although the random direction model addresses this problem, it requires that a border behavior be defined to guide mobility as the UE approaches the boundary. In this paper, a tracking area boundary-aware protocol for pseudo stochastic mobility prediction in LTE networks was designed, developed and simulated. In the developed protocol, the problem of boundary behavior for random direction mobility model has been addressed using CDF and received signal levels. The simulation results for UE coordinates, absolute angle, unit vector along this absolute angle, waypoints, velocity and pause times showed that this protocol is truly pseudo-stochastic. In addition, the developed protocol was observed to have abilities to detect when an UE has moved out of the current tracking area. Since LTE networks endeavor to attain lower latency compared to legacy cellular networks, the developed protocol is ideal for faster paging services that ultimately ensure high data rates. Future work lies on the investigation of the performance of the developed protocol in LTE in terms of bandwidth improvements. There is also need for further research on the applicability of this protocol for low latency vertical as well as horizontal handovers in cellular networks.

\section{References}

[1] Petteri N. (2016). Modeling Mobility. University of Helsinki. Pp. 1-47.

[2] Sani, Y., Isah, M., Edwards, C., \& Mauthe, A. (2017). Experimental evaluation of the impact of mobility management protocols on HTTP adaptive streaming. IET Networks, 6(6), 186-192.

[3] Patle V., \& Sanjay K. (2016). Evaluation of Mobility Model with MANET Routing Protocols. International Journal of Computer Applications. Volume 152 - No.8, pp. 8-12.

[4] Su, C. (2018). Vector Representation Based Model Considering Randomness of User Mobility for Predicting Potential Users. In PRIMA 2018: Principles and Practice of Multi-Agent Systems: 21st International Conference, Springer, Tokyo, Japan, October 29-November 2, Vol. 11224, p. 70.

[5] Tarik T., Abdelhakim H., \& Apollinaire N. (2011). Mobility-Aware Streaming Rate Recommendation System. Proceedings of IEEE Globecom.

[6] Priyan, M. K., \& Devi, G. U. (2018). Energy efficient node selection algorithm based on node performance index and random waypoint mobility model in internet of vehicles. Cluster Computing, 21(1), 213-227.

[7] Samuylov, A. K., Ivchenko, A. Y., Orlov, Y. N., Moltchanov, D. A., Bobrikova, E. V., Gaidamaka, Y. V., \& Shorgin, V. S. (2018). Kinetic Approach to Elasticity Analysis of D2D Links Quality Indicators Under Non-stationary Random Walk Mobility Model. In Internet of Things, Smart Spaces, and Next Generation Networks and Systems, pp. 224-235.

[8] Jogendra K., \& Panda M. (2016).Ad hoc network routing protocols on random waypoint model. IEEE.

[9] Steinbock, C., Biham, O., \& Katzav, E. (2017). Distribution of shortest path lengths in a class of node duplication network models. Physical Review, 96(3), 032301.

[10] Kulkarni, V., Moro, A., \& Garbinato, B. (2016). Mobidict: A mobility prediction system leveraging realtime location data streams. In Proceedings of the 7th ACM SIGSPATIAL International Workshop on GeoStreaming, pp. 1-10.

[11] Logambal R. \& Chitra K. (2016). Mobility models and their influence on mobile Ad Hoc Networks. Journal of Chemical and 
Pharmaceutical Sciences. Vol 9(4), pp. 1930-1934.

[12] Azevedo, C. L., Deshmukh, N. M., Marimuthu, B., Oh, S., Marczuk, K., Soh, H., \& Ben-Akiva, M. E. (2017). SimMobility Short-term: An integrated microscopic mobility simulator. Transportation Research Record, 2622(1), 13-23.

[13] Daniel H., Carolin T., Peter M., David W., Oliver H., Stefan R., André B. (2016). A hybrid and multiscale approach to model and simulate mobility in the context of public events. International Scientific Conference on Mobility and Transport Transforming Urban Mobility. Vol. (19), pp. 350-363.

[14] Fabrício A., Azzedine B., Thais R., Linnyer B., \& Antonio A. (2015). A novel macroscopic mobility model for vehicular networks. The International Journal of Computer and Telecommunications Networking. Vo. 79(C), pp. 188-202.

[15] Pokhrel, K., Dutta, N., Ghose, M. K., \& Sarma, H. K. D. (2020). Mathematical Modeling of Mobility Models used in IP-Based Wireless Mobile Networks. In Trends in Communication, Cloud, and Big Data, Springer, Singapore, pp. 21-29.

[16] Ahmad B. (2016). Hybrid fuzzy social mobility model. Karbala International Journal of Modern Science. Vol. 2(1), pp. 29-40.

[17] Kim B., Kyong H., \& Ki-I K. (2017).A Survey on Mobility Support in Wireless Body Area Networks. MDPI, pp.1-18.

[18] Mostafa K. (2016). User(s) Mobility Prediction in Mobile Networks to Enhance Location Based Services (LBS) Performance. Universitat Bern, pp. 1-25.

[19] Seung-Woo, K., Kaifeng, H., and Kaibin, H. (2018).Wireless Networks for Mobile Edge Computing: Spatial Modeling and Latency Analysis. arXiv, pp. 1-35.

[20] Mariano, G., André, P., Michele, T., \& Ciro, C. (2016). Predicting human mobility through the assimilation of social media traces into mobility models. Springer Open Journal, pp. 1-15.

[21] Mahmoud A., Ghaith H. \& Wail M. (2016). Improving Vertical Handoffs Using Mobility Prediction. International Journal of Advanced Computer Science and Applications. Vol. 7(3), pp 413-419.

\section{Authors' Profiles}

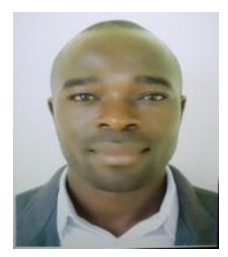

Vincent Omollo Nyangaresi, is a PhD student in the area of IT security and Audit. He has published numerous research articles covering areas such as communication systems, secure network communications, information systems acceptance modeling, TCP architecture and design, radio wave propagation, virtualization and cloud computing. In addition, he lecturers in the fields of computer networks, digital forensics, software engineering, information technology, and applied computer science.

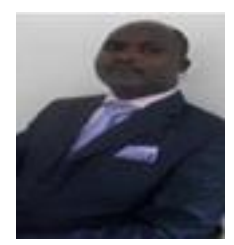

Dr. Silvance O. Abeka is currently the Dean, School Of Informatics And Innovative Systems, Jaramogi Oginga Odinga University of Science And Technology. He holds a masters degree in Business Administration (Information Technology) and a PhD in Management Information Science (MIS), from Kampala International University, Dar es Salaam Collage. His interests include Management Information Systems, Principles of Statistics and E- Commerce. He is also a lecturer in the school of Computer Studies.

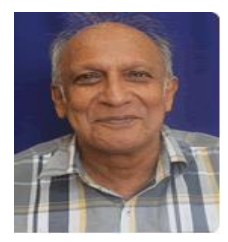

Prof. Anthony Joachim Rodriguez is a Kenyan computer scientist and educator. He holds a Bachelor of Science in electrical engineering (University of Manchester, England), Master of Science in control systems (University of Manchester, institute of science \& technology, England) and Doctor of philosophy in science computation (University of Manchester, institute of science \& technology, England). He has lectured in a number of universities and is currently a director, directorate of information and communications technology of Jaramogi Oginga Odinga university of science and technology. His research interests include scientific computation, approximation theory, modeling, informatics policy among others.

How to cite this paper: Vincent Omollo Nyangaresi, Silvance O. Abeka, Anthony J. Rodrigues, "Tracking Area Boundary-aware Protocol for Pseudo Stochastic Mobility Prediction in LTE Networks", International Journal of Information Technology and Computer Science(IJITCS), Vol.12, No.5, pp.52-62, 2020. DOI: 10.5815/ijitcs.2020.05.04 